WellBeing International

WBI Studies Repository

3-2007

\title{
Individual Acoustic Variation in Fallow Deer (Dama dama) Common and Harsh Groans: A Source-Filter Theory Perspective
}

\author{
Elisabetta Vannoni \\ University of Zurich \\ Alan G. McElligott \\ University of Zurich
}

Follow this and additional works at: https://www.wellbeingintlstudiesrepository.org/acwp_vsm

Part of the Animal Studies Commons, Comparative Psychology Commons, and the Other Animal

Sciences Commons

\section{Recommended Citation}

Vannoni, E., \& McElligott, A. G. (2007). Individual Acoustic Variation in Fallow Deer (Dama dama) Common and Harsh Groans: A Source-Filter Theory Perspective. Ethology, 113(3), 223-234.

This material is brought to you for free and open access by WellBeing International. It has been accepted for inclusion by an authorized administrator of the WBI Studies Repository. For more information, please contact wbisr-info@wellbeingintl.org.

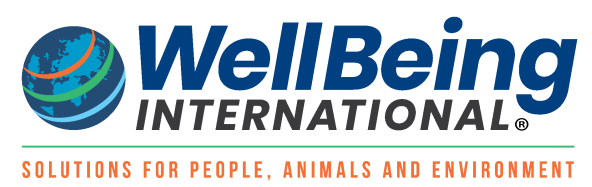




\title{
Individual Acoustic Variation in Fallow Deer (Dama dama) Common and Harsh Groans: A Source-Filter Theory Perspective
}

\author{
Elisabetta Vannoni and Alan G. McElligott \\ University of Zürich
}

\begin{abstract}
$\underline{\text { ABSTRACT }}$
Mammals are able to distinguish conspecifics based on vocal cues, and the acoustic structure of mammal vocalizations is directly affected by the anatomy and action of the vocal apparatus. However, most studies investigating individual patterns in acoustic signals do not consider a vocal production-based perspective. In this study, we used the source-filter model of vocal production as a basis for investigating the acoustic variability of fallow deer groans. Using this approach, we quantified the potential of each acoustic component to carry information about individual identity. We also investigated if cues to individual identity carry over among the two groan types we describe: common and harsh groans. Using discriminant function analysis, we found that variables related to the fundamental frequency contour and the minimum frequencies of the highest formants contributed most to the identification of a given common groan. Common groans were individually distinctive with $36.6 \%$ (53.6\% with stepwise procedure) of groans assigned to the correct individual. This level of discrimination is approximately six times higher than that predicted by chance. In addition, univariate ANOVAS showed significant inter-individual variation in the minimum formant frequencies when common and harsh groans were combined, suggesting that some information about individuality is shared between groan types. Our results suggest that the sound source and the vocal tract resonances act together to determine groan individuality and that enough variation exists to potentially allow individual recognition based on groans.
\end{abstract}

\section{Introduction}

The acoustic structure of vocalizations is usually variable and can be affected by factors such as species identity, individual identity, social context, and phenotypic traits of the vocalizing animal (Fischer et al. 2002; Gerhardt \& Huber 2002; Reby \& McComb 2003a; Blumstein \& Munos 2005). Individual variation in temporal and/or spectral features of acoustic signals is a pre-requisite for individual recognition. In situations in which vocal recognition is useful, selection may favour more stereotyped individual calls and the ability to perceive the discriminatory cues (Aubin et al. 2000; Insley 2001).

Individual vocal recognition may be adaptive for sexual selection, and influence male-male competition and female choice (Davies \& Halliday 1978; McComb 1991). The ability to distinguish between stranger and familiar males based on individually distinctive calls can help prevent unnecessary contests 
(Stoddard 1996). Moreover, females may become familiar with individual vocal differences and preferentially mate with familiar males who can afford higher energy courtship displays (East \& Hofer 1991; Zimmerman \& Lerch 1993). Therefore, it could be advantageous for vocalizing animals to broadcast information about individual identity.

Individual recognition based on vocal signatures is common in mammals (Rendall et al. 1996; Sayigh et al. 1998; Frommolt et al. 2003). The presence of individual characteristics in mammal vocalizations is not surprising because the individual morphology of their vocal apparatus directly influences the spectral structure of the emitted signal (Fitch \& Hauser 1995, 2003; Riede et al. 2005; McElligott et al. 2006). However, other than in humans (Homo sapiens), few studies have examined the individuality of vocalizations in relation to their biomechanical modes of production (Rendall et al. 1998; Soltis et al. 2005; Reby et al. 2006).

The source-filter theory of voice production (Fant 1960) predicts that the spectral structure of most mammal vocalizations results from the linear combination of two independent mechanisms. First, the source signal is generated by the vibration of the vocal folds during the passage of air in the glottis and characterizes the fundamental frequency (and its associated harmonics) of the vocalization. Then the source signal passes through the supra-laryngeal vocal tract where the resonance properties of the vocal tract cavities act as a filter and determine the amplification of specific frequencies called 'formants'. The formants finally radiate out through the mouth and nostrils into the environment. Because fundamental frequency and formant frequencies vary independently, one or both may provide receivers with information on caller identity (Rendall et al. 1998; Bachorowski \& Owren 1999).

Both sexes of some deer species are known to produce individually distinctive calls (Espmark 1974; Vaňková \& Málek 1997; Reby et al. 1998, 1999a; Torriani et al. 2006; Reby et al. 2006). The fallow deer is a highly polygynous and size dimorphic deer species (McElligott et al. 2001). While mature females may vocalize throughout the year using contact or alarm calls, mature males only produce their sexually selected groans during the breeding season and are otherwise silent. In the northern hemisphere, males start to vocalize towards the end of September and continue until early November. The vast majority of matings occur during the latter half of October and therefore some males begin to vocalize approximately 3 wk before the first matings occur (McElligott et al. 1999). The most common groan consists of a short, low-pitched and stereotyped vocalization characterized by a train of regularly spaced pulses (Reby et al. 1998). Vocalizations may be used by males to signal their presence to potential mates and to identify and localize competing males (McElligott \& Hayden 1999). Such a role for groaning suggests the existence of recognition cues for individuality. A previous study showed that fallow deer common groans contain information on individuality (Reby et al. 1998), but the factors responsible for inter-individual acoustic variation have not been explored in detail and the relative importance of different call features for achieving individuality remains unknown. Fallow deer males also produce a more noisy and harsh groan, and the acoustic structure of this call has never been described (E. Vannoni \& A. G. McElligott, personal observation). Because the production of various call types may rely on similar actions of the vocal folds and vocal tract of a specific individual, the acoustic cues to individuality originating from these processes may carry over across the vocal repertoire of the species (Rendall et al. 1998; Reby et al. 2006).

In this study, we investigated the individual acoustic variation of fallow buck groans in relation to the principles of the source-filter theory of sound production. We extracted and measured the spectral parameters of common and harsh groans and compared the formant pattern of the two types of call. Furthermore, we determined whether the acoustic structure of common groans varies according to individual identity, and the relative contribution of each feature in discriminating between different individuals. We finally determined if the acoustic individuality found in the formant pattern of common groans holds across harsh groans. 


\section{Methods}

\section{Study Site and Subjects}

The study was carried out on a herd of European fallow deer in Phoenix Park (709 ha, 80\% pasture, 20\% woodland; $53^{\circ} 22^{\prime} \mathrm{N}, 6^{\circ} 21^{\prime} \mathrm{W}$ ), Dublin, Ireland during five breeding seasons between 1997 and 2004. All males used in this study were of known age, had ear tags and were therefore individually distinguishable.

\section{Sound Recording and Signal Acquisition}

Vocalizations were recorded using a Sony digital audio tape recorder DAT-TCD D100 connected to a Sennheiser MKH 70 directional microphone. Recordings were performed between dawn and sunset when a male was groaning at a distance of $10-50 \mathrm{~m}$ from the microphone.

Fig. 1: Narrow band spectrograms of (a) a 'common' groan; and (b) a 'harsh' groan. The pulses and the first six formants are indicated

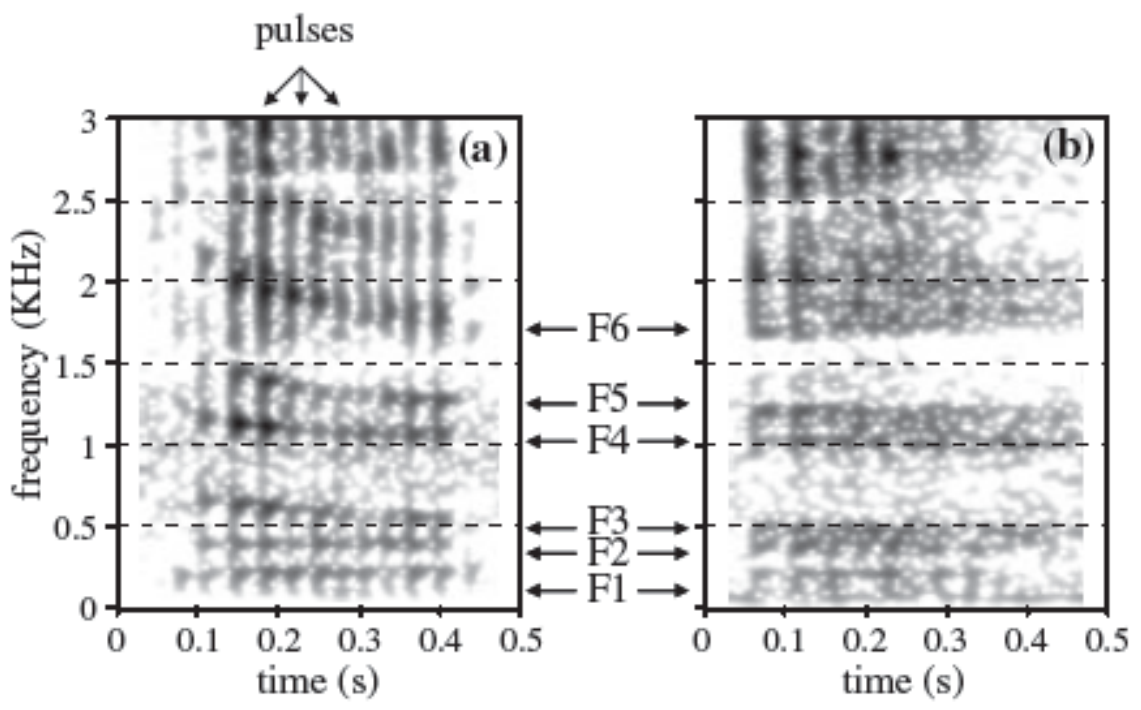

Vocalizations were transferred to a computer at a sampling frequency of $22.05 \mathrm{KHz}$ using Avisoft SASLab Pro 4.36 and saved in WAV format, at 16-bit amplitude resolution (Vannoni et al. 2005). As most of the recordings did not contain energy above $8 \mathrm{KHz}$, they were down-sampled to $16 \mathrm{KHz}$ for a better frequency resolution. Narrow-band spectrograms of common and harsh groans (Fig. 1, FFT method, window length $=0.03 \mathrm{~s}$, number of time steps $=1000$, number of frequency steps $=250$, frequency resolution $=20 \mathrm{~Hz}$, Gaussian window shape, dynamic range $=35 \mathrm{~dB}$ ) were generated using Praat 4.3.27 DSP package (P. Boersma \& D. Weenink, University of Amsterdam, Amsterdam, The Netherlands). Each groan was visually inspected and vocalizations with high levels of background noise were rejected.

During the breeding season, fallow deer males feed very little and lose approximately $26 \%$ of their body weight (McElligott et al. 2003), and there is some evidence from primates and deer that the acoustic structure of vocalizations can be affected by exhaustion (McComb 1988; Fischer et al. 2004). We therefore analysed recordings taken between October 9 and October 19 when only about $10 \%$ of the total number of matings had usually occurred (McElligott et al. 2001) and the majority of agonistic interactions 
among males were non-contact displacements (McElligott et al. 1998). This minimized the possibility that variation in the phonic structure of groans could have occurred due to exhaustion of the animals.

Fig. 2: Waveform of (a) a fallow deer common groan; and (b) its relative envelope. On the envelope of the signal, each amplitude peak is detected and indicated as 'pulse'

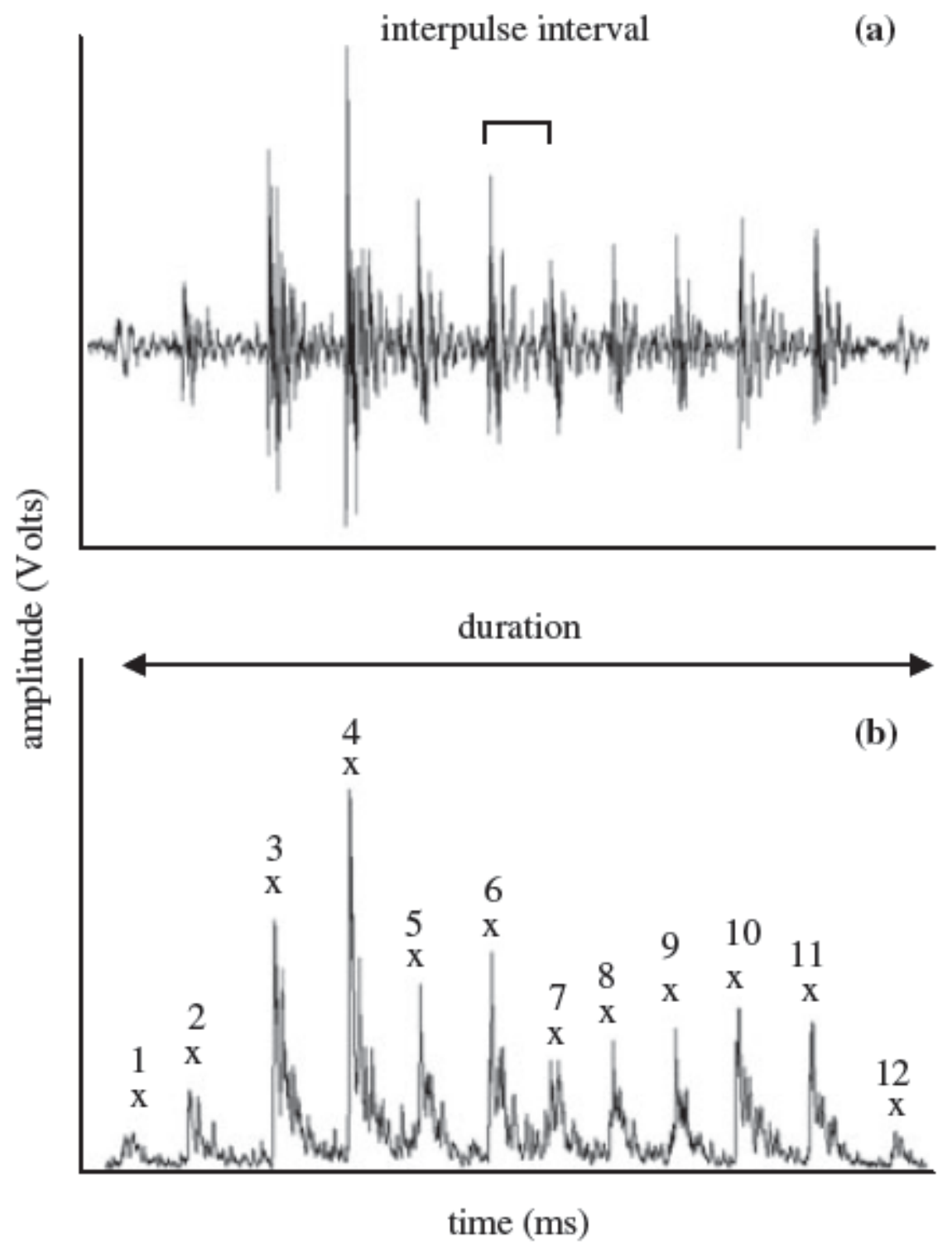

Vocalizations produced within the same bout are more likely to be acoustically homogeneous than vocalizations emitted in different bouts (Reby et al. 1999b), and therefore not statistically independent. To minimize this problem, the majority of the common groans (82\%) were randomly selected from different bouts and the remaining (18\%) groans belonged to long vocalization bouts but were never consecutive.

Acoustic Analyses

Common groans are short and low-pitched vocalizations, characterized by a train-pulse structure (Fig. 1a). The pulses represent the vibrations of the vocal folds and determine the fundamental frequency of 
the call. Fundamental frequency (F0) is defined as the inverse of the interpulse interval and this can be measured as the distance between consecutive pulse onsets (Fig. 2a). Distances between pulses were measured automatically from the envelope (amplitude vs. time) of the signal by using Pulse Train Analysis (Fig. 2b; method = rectification + exponential decay; pulse detection = peak search with hysteresis; time constant $=1 \mathrm{~ms}$; threshold $=0.1 \mathrm{~V}$; hysteresis $=16-19 \mathrm{~dB}$ ) in Avisoft SAS Lab-Pro 4.32. We calculated the values of the F0 along the groan and then averaged these values to obtain the mean $\mathrm{FO}\left(\mathrm{FO}_{\text {mean }}\right)$. Because all groans showed at least a modest frequency inflection, the minimum and the maximum $\mathrm{FO}\left(\mathrm{FO}_{\min }\right.$ and $\left.\mathrm{FO}_{\max }\right)$ were also included in the analysis. From the envelope of the signal we also calculated the number of pulses (pulses) and the duration of the groan (duration) as the distance between the onset of the first pulse and the end of the last one (Fig. 2b). Finally, we quantified the variation of F0 along the call using a measure of F0 perturbation, known as Jitter (Titze 1994). Jitter has been used as a measure of voice quality in mammals (Rendall 2003) and in a similar manner to other source-related features, may contribute to vocal distinctiveness (Owren \& Linker 1995). In this analysis, Jitter was calculated by dividing the average absolute difference between consecutive frequencies, by the mean F0 per groan (peak-picking method, Horii 1979; Titze et al. 1987).

In the spectrograms of common and harsh groans (Fig. 1), pronounced horizontal frequency bands are visible. These bands correspond to peaks in the spectrum and they can result from both the vibration pattern (regular or irregular) of the source and from the vocal tract (i.e. formant frequencies and nasal resonances). The conspicuous frequency bands of fallow deer groans represent formants (McElligott et al. 2006). The values of the first six formant frequencies (F1-F6) were estimated using Linear Predictive Coding analysis (LPC) [Sound: To Formant (burg) command] in Praat 4.3 DSP package. By performing a single LPC analysis on each groan, higher formants (F4-F6) were better detected and therefore more accurately measured than lower formants (F1-F3). We therefore conducted a double or a triple LPC analysis on each groan to get the best estimations of all formants. We first carried out an LPC analysis (time step $=0.01-0.02 \mathrm{~s}$, maximum number of formants $=3-4$, maximum formant $1 / 4700-850 \mathrm{~Hz}$, window length $0.1-0.26 \mathrm{~s}$ ) to measure the frequencies of the first three formants (F1-F3). Then, we performed a second LPC analysis (time step $=0.01-0.02 \mathrm{~s}$, maximum number of formants $=6-7$ maximum formant $=$ $1800-2600 \mathrm{~Hz}$, window length $0.1-0.26 \mathrm{~s}$ ) to estimate the last three formant frequencies (F4-F6). When the sixth formant was not detected by the second LPC analysis $(n=91)$, we conducted a third LPC analysis (time step $=0.01-0.02 \mathrm{~s}$, maximum number of formants $=5-6$ maximum formant $=1800-2600$ $\mathrm{Hz}$, window length $0.1-0.26 \mathrm{~s})$. LPC measurements were transferred to Excel. We calculated the minimum frequencies of the six formants $\left(F 1_{\min }-F 6_{\min }\right)$ from each groan by averaging the values over the last part of the call when formants become flat (plateau). We included in our analysis the minimum (and not the mean) formant frequencies because they are more likely to be related to individual characteristics (Fitch 1997; Reby \& McComb 2003a) and therefore to vary between individuals. We also estimated the minimum spacing of the formants [known as minimum formant dispersion $\left(\mathrm{Df}_{\min }\right)$ ] according to Reby \& McComb (2003a)

To examine the differences in the acoustic structure between common and harsh groans $(\mathrm{n}=41$ in both cases) we analysed and compared the two types of groans emitted from seven different males (4-8 calls for each individual, average $=6.4 \pm 0.75$ ). The same data set was also used to evaluate the intraindividual stability in the spectral structure across groan types. To investigate the individual-related variation in common groans, we constructed a larger data set required by this type of analysis; we analysed 153 groans from 16 different males $(7-10$ calls for individual, average $=9.6 \pm 0.24)$ ranging from 5 to 8 yr old. 


\section{Statistical Analysis}

We used the Wilcox on signed-ranks test to investigate the differences between the acoustic structure of common and harsh groans. We compared the averages of the minimum formant frequencies, the minimum formant dispersion and the maximum vocal tract length between the two types of groans.

We quantified the individual distinctiveness of the common groans by performing a principal component analysis (PCA) followed by a multivariate analysis of variance (MANOvA) and a discriminant function analysis (DFA). PCA and DFA require a full data matrix. Due to the different quality of the recordings, it was not possible with some groans $(n=35)$ to measure all the acoustic parameters and therefore our data set contained missing values. To preserve the full matrix $(n=153$ groans) we substituted missing values with the average of each variable (Blumstein \& Munos 2005). Moreover, we log transformed some of the acoustic variables (pulses, duration, $\mathrm{FO}_{\text {mean }}, \mathrm{FO}_{\min }$, and $\mathrm{FO}_{\max }$ ) to reach normal distributions. We used PCA to eliminate redundancy due to the high intercorrelation of the acoustic variables in our data set and to examine clustering among variables. PCA transforms a large number of correlated variables into a few orthogonal variables (principal components) that explain the maximum amount of variation in the original data set with a minimum loss of information. Principal components are weighted linear combinations of the original variables. We retained those components with eigenvalues greater than 1 (Kaiser's criterion) and applied a Varimax rotation of axes to improve component interpretation. The scores of the five extracted principal components were tested and confirmed for normality (KolmogorovSmirnov test) and used as input variables in the DFA.

We used a multivariate analysis of variance (mANOva) to confirm statistical differences in the principal component scores across individuals. We used DFA to quantify the extent to which individuals can be classified on the basis of their common groans and which group of variables account most for this classification (Johnson \& Wichern 1992). DFA identifies a linear combination of values (canonical functions) from two or more discriminating variables (component scores) that best separate cases (groans) into their a priori assigned discrete classes (individuals). On the basis of the discriminant functions, each groan was assigned to its appropriate group (correct assignment) or to another group (incorrect assignment). We calculated the percentage of correct classification due to chance by applying a randomization procedure. The expected level of correct assignment was averaged from DFAs performed on 1000 randomized permutations of the data set (Solow 1990; McGarigal et al. 2000). We cross-validated our results by performing a leave-one-out classification, an appropriate method for small sample sizes (Lachenbruch \& Mickey 1968; Goutte 1997; McGarigal et al. 2000).

Some principal component scores had similar factor loadings on the same discriminant function. Therefore, we conducted a second DFA to clarify the relative contribution of specific features in discriminating among individuals and to verify whether all the measured acoustic variables are important for individual separation. In this new analysis, we included all of the measured parameters and used a forward stepwise procedure (Johnson \& Wichern 1992). Variables were entered in this analysis based on the change in Wilk's lambda $(F$ to enter $=3.84 ; F$ to remove $=2.71)$.

To investigate whether formant-related information about individuality carry over across groan types, we examined if male common and harsh groans, considered as a set, were consistently different between individuals. For this purpose, we first built a sample with common and harsh groans combined as they represented a single call type. Then, we conducted a univariate analysis of variance (ANOVA) on each of the seven variables considered $\left(F 1_{\min }-F 6_{\min }\right.$ and $\left.D f_{\min }\right)$ to test for between-male differences. We used Kolmogorov-Smirnov test to determine if the variables were normally distributed. The randomization test was performed using R 2.0.1. (R Development Core Team 2004). All the other statistical analyses were 
conducted using SPSS 11.5. All tests were two-tailed and factors were considered to have a statistically significant influence if $p<0.05$. All means are given with standard errors.

\section{Results}

\section{Acoustic Characteristics of Common and Harsh Groans}

Common groans are characterized by a pulse-train structure (Fig. 1a). Groans lasted from 230 to $607 \mathrm{~ms}$ $(\mathrm{n}=153$; mean $=383.0 \pm 6.1 \mathrm{~ms})$ and contained a number of pulses, ranging from 7 to 18 per groan $(\mathrm{n}=$ 153; mean $=11.0 \pm 0.2$ ). The $F 0_{\text {mean }}$ was low and varied from 21 to $39 \mathrm{~Hz}(\mathrm{n}=153$; mean $=28.2 \pm 0.3$ $\mathrm{Hz})$. The $\mathrm{FO}_{\text {min }}$ ranged from 16 to $29 \mathrm{~Hz}(\mathrm{n}=153$; mean $=22.3 \pm 0.2 \mathrm{~Hz})$ while the $\mathrm{FO}_{\max }$ varied from 22 to $55 \mathrm{~Hz}(\mathrm{n}=153$; mean $=34.7 \pm 0.5)$. The variability of the fundamental frequency along the call was quantified by the jitter parameter, which ranged from 2.0 to $29.6 \%(n=153$; mean $=12.6 \pm 0.4 \%)$. There were six formants within the first $2.5 \mathrm{KHz}$ of frequency of the common groans. The first two formants are flat over the whole groan. The F1min ranged from 152 to $263 \mathrm{~Hz}(\mathrm{n}=134$; mean $=208.5 \pm 2.0 \mathrm{~Hz})$ whereas the $\mathrm{F} 2$ min ranged from 329 to $496 \mathrm{~Hz}(\mathrm{n}=138$; mean $=414.3 \pm 1.9 \mathrm{~Hz})$. From the third formant upwards, frequencies decrease along the call until they reach a minimum value at the plateau. The minimum frequency ranged from 457 to $677 \mathrm{~Hz}$ for $\mathrm{F} 3(\mathrm{n}=149$; mean $=575.2 \pm 3.3 \mathrm{~Hz}$ ), from 966 to $1149 \mathrm{~Hz}$ for $\mathrm{F} 4(\mathrm{n}=153$; mean = $1060.2 \pm 2.9 \mathrm{~Hz})$, from 1170 to $1371 \mathrm{~Hz}$ for $\mathrm{F} 5(\mathrm{n}=153$; mean $=1265.9$ $\pm 3.2 \mathrm{~Hz})$, and from 1672 to $1974 \mathrm{~Hz}$ for $\mathrm{F} 6(\mathrm{n}=147$; mean $=1806.9 \pm 5.2 \mathrm{~Hz})$. The $\mathrm{Df}_{\text {min }}$ varied from 281 to $326 \mathrm{~Hz}(\mathrm{n}=132$; mean $=300.6 \pm 0.8 \mathrm{~Hz})$.

Harsh groans are typically emitted in a short series, preceded and followed by common groans. They constitute most of the post-copulatory vocalizations (McElligott \& Hayden 2001) and they are also often associated with marking behaviour (E. Vannoni, personal observation). The F0 of harsh groans is not well defined (Fig. 1b) and while two to three pulses are usually visible at the beginning of the groan, the pulsetrain structure disappears. The formants are clear and are usually flat or show a weak modulation over the length of the groan. The minimum frequencies of the last four formants $\left(F 3_{\min }-F 6_{\min }\right)$ of the harsh groans were significantly different than in common groans (Table 1). However, the minimum formant dispersion did not differ between the two types of groans.

Table 1: Acoustic parameters measured on common and harsh groans $(n=41$ for both) from males $(n=7)$ that provided both types of groans

\begin{tabular}{|c|c|c|c|c|c|c|}
\hline \multirow[t]{2}{*}{ Acoustic parameter } & \multicolumn{2}{|c|}{ Common groans } & \multicolumn{2}{|c|}{ Harsh groans } & \multirow[b]{2}{*}{$\mathbf{p}$} & \multirow[b]{2}{*}{$\mathbf{z}$} \\
\hline & Mean & SE & Mean & SE & & \\
\hline $\mathrm{F} 1_{\min }(\mathrm{Hz})$ & 211.22 & 3.05 & 199.36 & 6.05 & 0.13 & -1.52 \\
\hline $\mathrm{F} 2{ }_{\min }(\mathrm{Hz})$ & 412.83 & 5.80 & 416.20 & 4.58 & 0.40 & -0.85 \\
\hline $\mathrm{F} 3_{\min }(\mathrm{Hz})$ & 570.67 & 5.70 & 514.72 & 5.57 & 0.02 & -2.37 \\
\hline $\mathrm{F} 4_{\min }(\mathrm{Hz})$ & 1055.03 & 10.27 & 1079.42 & 12.58 & 0.03 & -2.20 \\
\hline $\mathrm{F} 55_{\min }(\mathrm{Hz})$ & 1253.37 & 9.53 & 1213.98 & 11.84 & 0.03 & -2.20 \\
\hline $\mathrm{F} 6_{\min }(\mathrm{Hz})$ & 1791.09 & 24.82 & 1851.82 & 21.59 & 0.03 & -2.20 \\
\hline $\mathrm{Df}_{\min }(\mathrm{Hz})$ & 298.49 & 3.03 & 302.58 & 2.54 & 0.24 & -1.19 \\
\hline VTLmax $(\mathrm{cm})$ & 58.67 & 0.59 & 57.86 & 0.48 & 0.24 & -1.19 \\
\hline
\end{tabular}

Results from the pairwise comparison (Wilcoxon matched-pairs signed-rank test) showed a significant difference in the minimum frequencies of the last four formants between common and harsh groans. 


\section{Acoustic Cues to Identity}

Five components (PC1-PC5) that exceeded Kaiser's criterion (eigenvalues greater than 1) were generated from the PCA performed on the common groans $(n=16$, Table 2). These five components combined accounted for $82 \%$ of the variation in the original data set. Filter-related parameters were grouped in two different components (PC1 and PC4) that reflect different aspects of the vocal tract function. PC1 included higher formant frequencies $\left(F 3_{\mathrm{min}}-F 6_{\min }\right)$, which are mainly related to the length of the vocal tract, whereas PC4 consisted of lower formants $\left(F 1_{\min }-F 3_{\min }\right)$ generally associated to the shape of the vocal tract. Two components reflected aspects of source characteristics; PC2 represented

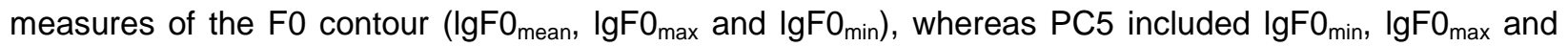
the Jitter, a measure of voice quality based on the stability of vocal folds vibration. Finally, temporal parameters (IgPulses and IgDuration) were highly correlated with PC3. Groans were significantly different among males for all principal component scores (manova: PC1, $F_{15,137}=10.47 ; P C 2, F_{15,137}=14.76 ; P C 3$, $F_{15,137}=2.90 ; P C 4, F_{15,137}=5.14 ; P C 5, F_{15,137}=6.04 ; p<0.001$ in all cases).

Cross-validated DFA classified $36.6 \%$ of the male common groans correctly. The mean percentage of correct assignment due to chance calculated by the randomization test was $6.19 \pm 0 \%$. Because none of the 1000 data sets had a correct classification greater than or equal to the original data set, groans were correctly classified at a percentage higher than that expected by chance at $p<0.001$. Five significant discriminant functions were generated (Ps $<0.01$, Table 3). The first two discriminant functions together accounted for $81.1 \%$ of the variation and were correlated with scores from the PC1 and PC2. The third discriminant function accounted for another $10.6 \%$ and was correlated with scores from PC3, PC4 and PC5. The remaining two discriminant functions were residual in nature, but combined explained $8.3 \%$ of the variance.

Table 2: Rotated factor loadings of the measured acoustic variables on the five PCs with eigenvalues $>1$

\begin{tabular}{|c|c|c|c|c|c|}
\hline \multirow[b]{2}{*}{ Acoustic variable } & \multicolumn{5}{|c|}{ Components } \\
\hline & 1 & 2 & 3 & 4 & 5 \\
\hline $\mathrm{Df}_{\min }$ & 0.95 & 0.03 & -0.04 & 0.17 & 0.06 \\
\hline $\mathrm{F} 6_{\text {min }}$ & 0.91 & 0.04 & -0.06 & 0.00 & 0.05 \\
\hline $\mathrm{F} 5_{\text {min }}$ & 0.88 & 0.01 & -0.01 & 0.15 & -0.05 \\
\hline $\mathrm{F} 4_{\text {min }}$ & 0.86 & 0.04 & 0.09 & -0.19 & 0.02 \\
\hline $\operatorname{lgF} 0_{\text {mean }}$ & 0.01 & 0.96 & -0.01 & -0.02 & 0.09 \\
\hline $\operatorname{lgF} 0_{\max }$ & 0.01 & 0.83 & 0.08 & 0.02 & 0.44 \\
\hline $\operatorname{lgFO_{\text {min}}}$ & 0.12 & 0.78 & -0.10 & -0.05 & -0.49 \\
\hline IgPulses & -0.00 & 0.29 & 0.95 & -0.02 & 0.00 \\
\hline IgDuration & -0.00 & -0.31 & 0.94 & -0.01 & 0.01 \\
\hline $\mathrm{F} 1_{\min }$ & -0.33 & -0.08 & 0.07 & 0.77 & 0.04 \\
\hline $\mathrm{F} 3_{\text {min }}$ & 0.28 & -0.19 & -0.13 & 0.67 & -0.23 \\
\hline $\mathrm{F} 2$ min & 0.17 & 0.20 & 0.01 & 0.57 & 0.21 \\
\hline Jitter & 0.07 & 0.10 & -0.01 & 0.93 & 0.92 \\
\hline Eigenvalue & 3.48 & 2.49 & 1.82 & 1.46 & 1.41 \\
\hline$\%$ Variance explained & 26.77 & 19.16 & 14.03 & 11.21 & 10.81 \\
\hline
\end{tabular}

Bold types indicates the heaviest factor loadings; a cutoff point of \pm 0.40 was used to include a variable in a component. The eigenvalues and their explained variance of each component are given at the bottom of the table. 
Not all the acoustic variables significantly contributed to the assignment of common groans to the correct individuals, when they were used in the stepwise DFA. Seven variables entered the analysis: four formant frequencies $\left(\mathrm{F} 2_{\min }, \mathrm{F} 3_{\min }, \mathrm{F} 5_{\min }\right.$ and $\left.\mathrm{F} 6_{\min }\right)$, two $\mathrm{F} 0$-related parameters $\left(\mathrm{IgFO} 0_{\min }\right.$ and $\left.\mathrm{lgF} \mathrm{O}_{\text {mean }}\right)$ and one temporal parameter (IgDuration). The IgFOmin entered the analysis first followed by the $\mathrm{F} 6_{\text {min }}$ and the $\lg \mathrm{F}_{\text {mean. }}$. The cross-validated percentage of correct assignment was of $53.6 \%$.

When common and harsh groans were considered as a single call type, the univariate analyses of variance indicated significant heterogeneity among individuals for most of the formant frequencies and for the minimum formant dispersion (ANOVAS: $F 1_{\text {min }}, F_{6,75}=1.69, p=0.14 ; F 2_{\text {min }}, F_{6,75}=5.75, p<0.01 ; F 3_{\min }$, $F_{6,75}=0.22, p=0.97 ; F 4_{\min }, F_{6,75}=6.69, p<0.01 ; F 5_{\min }, F_{6,75}=2.57, p<0.05 ; F 6_{\min }, F_{6,75}=11.53, p<$ $\left.0.01 ; \mathrm{Df}_{\min }, \mathrm{F}_{6,75}=7.78, \mathrm{p}<0.01\right)$.

Table 3: Structure matrix from the DFA, conducted using the five PC scores. The matrix shows the correlations between the PC scores and the discriminant functions and therefore the relative contribution of each variable to the separation of individuals

\begin{tabular}{|lccccc|}
\hline & \multicolumn{5}{c|}{ Functions } \\
\cline { 2 - 6 } PC scores & $\mathbf{1}$ & $\mathbf{2}$ & $\mathbf{3}$ & $\mathbf{4}$ & $\mathbf{5}$ \\
\hline PC1 & $\mathbf{0 . 4 7}$ & $\mathbf{0 . 4 5}$ & -0.07 & $\mathbf{- 0 . 6 3}$ & -0.42 \\
PC2 & $\mathbf{0 . 6 0}$ & $-\mathbf{0 . 5 0}$ & -0.26 & 0.37 & 0.42 \\
PC3 & 0.03 & 0.06 & $\mathbf{0 . 4 6}$ & -0.41 & $\mathbf{0 . 7 9}$ \\
PC4 & 0.14 & 0.32 & $\mathbf{0 . 5 1}$ & $\mathbf{0 . 7 8}$ & -0.05 \\
PC5 & -0.05 & 0.41 & $-\mathbf{0 . 6 6}$ & 0.24 & $\mathbf{0 . 5 7}$ \\
Eigenvalue & 2.95 & 1.75 & 0.61 & 0.26 & 0.22 \\
\% Variance explained & 50.90 & 30.20 & 10.60 & 4.50 & 3.80 \\
\hline
\end{tabular}

Bold types indicates correlations greater that $r=0.45$.

\section{Discussion}

We found that the fundamental frequency-related parameters and minimum frequencies of the highest formants accounted for $>80 \%$ of the variation, in the individually distinctive common groans of fallow bucks. It was possible to correctly classify the groans of 16 individuals in $36.6 \%$ of cases, and in $53.6 \%$ of cases using the stepwise procedure. Minimum formant frequencies still showed significant inter-individual variation when common and harsh groans were combined, indicating that some information about individuality holds across groan types. Studies investigating vocal individuality in mammals such as humans and other primates, have shown the prominent role of vocal tract resonances as cues to individual identity (Owren et al. 1997; Bachorowski \& Owren 1999). Our results suggest that in fallow deer, source and filter-related features contribute equally to the vocal identity, possibly because of different selection pressures acting on the acoustic structure of groans.

Common groans were characterized by a series of low-frequency glottal pulses produced by the larynx. Pulses contain broadband energy and therefore emphasize the resonances of the vocal tract (Fitch 1997; Riede \& Zuberbühler 2003). In fallow deer, these resonances are likely to convey important information such as the body size of the caller (Fitch \& Reby 2001; McElligott et al. 2006). The production of pulsetrain vocalizations may therefore have evolved to broadcast formant-related information in a more efficient way. However, formant frequencies play a crucial role in the vocal communication of red deer (Cervus elaphus), in which males mainly produce vocalizations characterized by a harmonic structure, sometimes interrupted by non-linear phenomena, and with higher F0 than that of groans (Reby \& 
McComb 2003a; Reby et al. 2005). The extremely low F0 and the pulse structure of fallow deer groans suggest a similar production mechanism to that of the human pulsed phonation (Blomgren et al. 1998). This phonation mode is characterized by reduced subglottal pressure (associated with the expiratory force) and lower airflow than other phonation types (Blomgren et al. 1998). Pulse signals are therefore physiologically easy to produce (Riede \& Zuberbühler 2003) and may require less effort from the vocalizing individual. Fallow bucks commonly groan at rates of 60 per minute and can also groan at rates of 90 for very short periods (McElligott \& Hayden 1999, 2001). Such high vocalization rates not only play a role in intrasexual competition, but are also probably necessary to facilitate the detection and the recognition of the information contained in the vocalizations in a situation where many conspecifics vocalize in close proximity (Jouventin et al. 1999; McElligott \& Hayden 1999). We suggest that the production of pulsed vocalizations could be adaptive if this would allow males to save energy and therefore to achieve and sustain high vocalization rates. However, data on the aerodynamics and physiological properties of the vocal production mechanism in fallow deer are needed to test this hypothesis.

Formants showed a weak modulation along the harsh groans in a similar manner to red deer harsh roars. This suggests a static posture of the vocalizing male and the onset of the vocalization when the larynx is already pulled down towards the sternum (Reby \& McComb 2003b; McElligott et al. 2006; this study). The observed differences in formant values between common and harsh groans may represent differences in the vocalization posture or articulatory movements (movement of the tongue, mandible, and lips) during the production of the two types of groans (Rendall et al. 1998). However, there was no significant difference in the minimum formant dispersion between the two types of groans, suggesting a similar extent of laryngeal lowering.

Discriminant function analyses confirmed that fallow deer common groans are individually distinctive. The percentage of correct assignment (36.6\%) was significantly higher than that expected by chance (6.3\%), but lower than that previously reported for fallow deer groans (87.9\% using network classification; Reby et al. 1998). This discrepancy is probably due to the larger number of individuals used in our analysis and for statistical reasons, this results in a lower classification rate. Moreover, it is important to consider that our result represents a conservative estimate of the percentage of correct assignment. The loss of about $20 \%$ of the variance in the acoustic properties due to the use of the PCA probably reduced the number of groans that were assigned to the correct individuals. This hypothesis is supported by the higher percentage of correct assignment that we obtained from the stepwise DFA (53.6\%), in which the acoustic parameters (and not the principal component scores) were used, and all available information was therefore preserved. Generally, with mammal vocalizations, the variety of acoustic parameters included in DFA and the diverse sample sizes used to examine the degree of individuality, make it difficult to compare the absolute values of correct assignment across studies. Nevertheless, we found that calls were assigned to the correct individual with a probability of 6 (DFA with PC scores) to 8.5 (stepwise DFA) times higher than that predicted by chance, a result similar to that reported for other mammals such as baboons (Owren et al. 1997; Fischer et al. 2002).

The examination of the structure matrix of the DFA (Table 3) indicates that characteristics associated with source energy, vocal tract filter, and temporal features, all contribute to the identification of a given common groan, and with variables related to the F0 contour and the minimum frequencies of the higher formants being the most distinctive. This finding is consistent with those on acoustic individuality in African elephants (Loxodonta africana), baboons (Papio hamadrayas ursinus), and humans showing that both the vocal folds and supra-laryngeal structures play important roles in shaping the call and producing inter-individual acoustic differences (Bachorowski \& Owren 1999; Rendall 2003; Soltis et al. 2005). However, we found that variation in the vocal-tract resonances did not provide more stable, individually 
distinctive acoustic information than variation in vocal fold activity. Instead, the similar values of the correlations between the scores of PC1 and PC2 and the first two discriminant functions (Table 3) indicate a comparable contribution of filter-related characteristics (especially those related to the vocal tract length) and source-related characteristics in differentiating individuals, with the latter being slightly more important. Nonetheless, filter-related features that were not measured in this study, such as formant amplitudes and spectral shape, could also contribute to vocal distinctiveness (Rendall 2003) and lead to a larger influence of vocal tract resonances on acoustic individuality.

Results from the stepwise DFA indicated that the discrimination power of the component in which variables of the $\mathrm{FO}$ contour were included, is mainly determined by the minimum $\mathrm{FO}\left(\mathrm{FO}_{\mathrm{min}}\right)$, followed by the mean $\mathrm{FO}\left(\mathrm{FO}_{\text {mean }}\right)$. $\mathrm{FO}_{\text {min }}$ represents the minimum rate at which the vocal folds vibrate and this is physiologically constrained by their length (Titze 1994; Fitch 1997). It is therefore not surprising that the $\mathrm{FO}_{\text {min }}$ is the acoustic feature that varies most among individuals. Pulsed vocalizations generally show a narrow range of variation of F0 (Blomgren et al. 1998; Riede \& Zuberbühler 2003; Frey et al. 2006). This suggests a highly stabilized vibration pattern, which is likely to contribute to the stability of the F0-related parameters within individuals. However, sexual selection might have favoured the evolution of vocal cues to the fitness-related characteristics in fallow deer males, and led to the selection of lower pitch vocalizations, indicating higher-quality individuals. Such low pitch may persist and be perceived at distances from the caller at which formants are likely to be distorted (O'Connell-Rodwell et al. 2000; McComb et al. 2003).

Although all filter-related characteristics potentially represent cues to caller identity, lower formants contribute less than higher ones to the inter-individual variability of the acoustic structure of common groans. One explanation based on what is known about human vocal production is that while lower formants are determined by changes in the shape of the vocal tract occurring continuously during vocalization, higher formants may be less variable because they represent the resonance effects that are primarily determined by the length of the vocal tract (Fant 1960; Reby \& McComb 2003a). However, the extent to which the vocal production mechanism in fallow deer (especially in terms of vocal tract shape and vocal folds dynamics) is similar to that of humans remains to be investigated. In our study, the frequency of the highest formant $\left(F 6_{\text {min }}\right)$ entered the stepwise analysis first and this was the variable that most strongly related to the minimum formant dispersion $\left(\mathrm{Df}_{\mathrm{min}}\right)$. Formant dispersion is related to vocal tract length and in turn to the body size of a variety of mammals (Fitch 1997; Riede \& Fitch 1999; Reby \& McComb 2003a). Therefore, individual variation in formants might be traced to some extent to differences in body size between individuals.

Other factors related to the source energy (Jitter) and temporal pattern are likely to play a role in individual vocal distinctiveness in fallow deer common groans, because the scores of their component (PC3 and PC5, respectively) are correlated with the third discriminant function (Table 3). Results of the stepwise DFA showed that the duration of a groan explained a small percentage of inter-individual variation in the acoustic structure and contributed to improved classification accuracy. In contrast, Jitter did not enter the analysis and therefore appears to have a marginal (if any) role in determining vocal individuality. However, because of the variety of methods that can be used to calculate Jitter (reviewed in Titze \& Liang 1993), these results are only applicable to our method of measuring Jitter. Although Jitter may not differ substantially between individuals, it represents a measure of vocal perturbation and may therefore provide information about arousal states (Gamba \& Giacoma 2005; Riede et al. 2005).

Individual characteristics of vocalizations can be specific to each call type or can be shared by several call types. The acoustic structure of the harsh groans mainly consists of broadband energy determined by irregular vibrations of the vocal folds. This non-periodic energy source generates noise that in the spectrum looks like deterministic chaos (Wilden et al. 1998). While the presence of this non-linear 
phenomenon in calls of certain individuals (and not of others) may provide an additional cue to individuality (Fitch et al. 2002), it makes all the information-related to the F0 unavailable to receivers. Formants have been hypothesized to represent a highly stable and reliable source of information about the identity of the caller carrying this information across a variety of vocalization contexts and call types (Owren et al. 1997). Results from our univariate analysis of variance performed on a combined set of harsh and common groans indicated that the vocal tract resonances of the two types of groans share cues to identity. Fallow deer might therefore possess individual voices as shown in other mammals (humans, Doddington 1985; rhesus monkeys, Macaca mulatto, Rendall et al. 1998, red deer, Reby et al. 2006). However, it is unlikely that these cues alone allow individual recognition because of the large contribution to individuality of the source-related characteristics.

Bouts of harsh groans are more likely to be delivered when other males are close to the vocalizing individual (E. Vannoni, personal observation) and therefore when other sensory channels (such as vision or olfaction) are available for recognition. In this context, it could be more beneficial for males to produce vocalizations with non-periodic energy sources, such as harsh groans, in which the information about individuality is reduced (Rendall et al. 1998) but other formant-related information such as body size is emphasized (Fitch et al. 2002; Reby et al. 2005). Morton (1977) suggested that harshness is tied to large body size indirectly through the production of low-pitched sounds. However, in homoeothermic vertebrates, size at maturity is constant and therefore the use of low or high frequency sounds might be more important in conveying information about motivation, than about body size (Morton 1982). In mammals, although harshness is generally associated with high subglottal pressure and therefore likely to reflect the motivational state of the caller (Reby \& McComb 2003b), it is also characterized by broadband energy that enhances the transmission of body size information related to formants (Fitch et al. 2002; Reby et al. 2005).

The phonic structure of fallow buck groans differs greatly from the naturally selected contact calls of adult female fallow deer. Adult females mainly produce tonal calls with relatively high fundamental frequency, and calls composed of a first noisy part and a second part with harmonic structure (Torriani et al. 2006). Some female calls are characterized by a low pitch and a noisy structure. In these, a small decrease in formants is evident along the call, indicating a small vocal tract elongation due to the lowering of the larynx (McElligott et al. 2006; Torriani et al. 2006). The fundamental and formant frequencies of female calls are considerably higher than those of male groans. This is not surprising given the sexual size dimorphism (McElligott et al. 2001), and therefore females have shorter vocal folds and vocal tract. Moreover, the sterno-thyroid and sterno-hyoid muscles, which allow the descent of the larynx towards the sternum, are highly sexually dimorphic (Reby \& McComb 2003b).

To conclude, this is the first time that the individually distinct acoustic structure of fallow deer common groans was examined from a source-filter theory perspective. Our results suggest that formant frequencies produced by the filtering process in the vocal tract and acoustic characteristics related to voice source, play similar roles in coding for individuality. This study also provides the first quantitative description of the acoustic structure of harsh groans, and suggests that this structure carries some of the information about individuality. Whether the variation in the acoustic cues to individuality are used by the animals for individual recognition remains to be investigated. However, given that fallow deer fawns can identify their mothers based on their individually distinctive vocalizations (Torriani et al. 2006), then it is also likely that adults can identify individuals when acoustic cues to individuality are available in calls.

\section{Acknowledgements}

We thank Tom Hayden, Billy Clarke and others at University College Dublin for their assistance. We thank Dúchas The Heritage Service (Ireland), and the staff of Phoenix Park for their support. Thanks to Chris 
Darwin and David Reby for advice during preparation of the manuscript. We are grateful to Linda Hollén, Hansjörg Kunc, Marco Torriani and the reviewers for their comments. We acknowledge the financial support of the Forschungskommission der Universität Zürich, and the Swiss Academy of Sciences to E. Vannoni.

\section{Literature Cited}

Aubin, T., Jouventin, P. \& Hildebrand, C. 2000: Penguins use the two-voice system to recognize each other. Proc. R. Soc. Lond. B. 267, 1081-1087.

Bachorowski, J.-A. \& Owren, M. J. 1999: Acoustic correlates of talker sex and individual talker identity are present in a short vowel segment produced in running speech. J. Acoust. Soc. Am. 106, 10541063.

Blomgren, M., Chen, Y., Ng, M. L. \& Gilbert, H. R. 1998: Acoustic aerodynamic, physiologic, and perceptual properties of modal and vocal fry registers. J. Acoust. Soc. Am. 103, 2649-2658.

Blumstein, D. T. \& Munos, O. 2005: Individual, age and sex-specific information is contained in yellowbellied marmot alarm calls. Anim. Behav. 69, 353-361.

Davies, N. B. \& Halliday, T. R. 1978: Deep croaks and fighting assessment in toads (Bufo bufo). Nature 274, 683-684.

Doddington, G. 1985: Speaker recognition-identifying people by their voice. Proceedings of the IEEE 73, 1651-1662.

East, M. L. \& Hofer, H. 1991: Loud calling in a female-dominated mammalian society: II. Behavioral contexts and functions of whooping of spotted hyaenas, Crocuta crocuta. Anim. Behav. 42, 651669.

Espmark, Y. 1974: Individual characteristics in the calls of reindeer calves. Behaviour 54, 50-59.

Fant, G. 1960: Acoustic Theory of Speech Production. The Hague, Mouton.

Fischer, J., Hammerschmidt, K., Cheney, D. L. \& Seyfarth, R. M. 2002: Acoustic features of male baboon loud calls: influences of context, age, and individuality. J. Acoust. Soc. Am. 111, 1465-1474.

Fischer, J., Kitchen, D. M., Seyfarth, R. M. \& Cheney, D. L. 2004: Baboon loud calls advertise male quality: acoustic features and their relation to rank, age and exhaustion. Behav. Ecol. Sociobiol. $56,140-148$.

Fitch, W. T. 1997: Vocal tract length and formant frequency dispersion correlate with body size in rhesus macaques. J. Acoust. Soc. Am. 102, 1213-1222.

Fitch, W. T. \& Hauser, M. D. 1995: Vocal production in nonhuman primates: acoustics, physiology, and functional constraints on "honest" advertisement. Am. J. Primatol. 37, 191-219.

Fitch, W. T. \& Hauser, M. D. 2003: Unpacking "Honesty": vertebrate vocal production and the evolution of acoustic signal. In: Acoustic Communication. (Simmons, A. M., Popper, A. N. \& Fay, R. R., eds). Springer, New York, pp. 65-137.

Fitch, W. T. \& Reby, D. 2001: The descended larynx is not uniquely human. Proc. R. Soc. Lond. B. 268, 1669-1675.

Fitch, W. T., Neubauer, J. \& Herzel, H. 2002: Calls out of chaos: the adaptive significance of nonlinear phenomena in mammalian vocal production. Anim. Behav. 63, 407-418.

Frey, R., Gebler, A. \& Fritsch, G. 2006: Arctic roars-Laryngeal anatomy and vocalization of the musk ox (Ovibos moschatus Zimmermann, 1780, Bovidae). J. Zool. (Lond.) 268, 433-448.

Frommolt, K. H., Goltsman, M. E. \& Macdonald, D. W. 2003: Barking foxes, Alopex lagopus: field experiments in individual recognition in a territorial mammal. Anim. Behav. 65, 509-518.

Gamba, M. \& Giacoma, C. 2005: Key issues in the study of primate acoustic signals. J. Anthropol. Sci. 83, 61-87.

Gerhardt, H. C. \& Huber, F. 2002: Acoustic Communication in Insects and Anurans: Common Problems and Diverse Solutions. University Chicago Press, Chicago, IL. 
Goutte, C. 1997: Note on free lunches and cross-validation. Neural Comput. 9, 1211-1215.

Horii, Y. 1979: Fundamental frequency perturbation observed in sustained phonation. J. Speech Hear. Res. 22, 5-19.

Insley, S. J. 2001: Mother-offspring vocal recognition in northern fur seals is mutual but asymmetrical. Anim. Behav. 61, 129-137.

Johnson, R. A. \& Wichern, D. W. 1992: Applied Multivariate Statistical Analysis. Prentice-Hall, Englewood Cliffs, NJ.

Jouventin, P., Aubin, T. \& Lengagne, T. 1999: Finding a parent in a king penguin colony: the acoustic system of individual recognition. Anim. Behav. 57, 1175-1183.

Lachenbruch, P. A. \& Mickey, M. R. 1968: Estimation of error rates in discriminant analysis. Technometrics 10, 1-111.

McComb, K. E. 1988: Roaring and reproduction in red deer, Cervus elaphus. PhD Thesis. University of Cambridge, Cambridge, UK.

McComb, K. E. 1991: Female choice for high roaring rates in red deer, Cervus elaphus. Anim. Behav. 41, 79-88.

McComb, K. E., Reby, D., Baker, L., Moss, C. \& Sayialel, S. 2003: Long-distance communication of acoustic cues to social identity in African elephants. Anim. Behav. 65, 317-329.

McElligott, A. G. \& Hayden, T. J. 1999: Context-related vocalization rates of fallow bucks, Dama dama. Anim. Behav. 58, 1095-1104.

McElligott, A. G. \& Hayden, T. J. 2001: Postcopulatory vocalizations of fallow bucks: who is listening? Behav. Ecol. 12, 41-46.

McElligott, A. G., Mattiangeli, V., Mattiello, S., Verga, M., Reynolds, C. A. \& Hayden, T. J. 1998: Fighting tactics of fallow bucks (Dama dama, Cervidae): reducing the risks of serious conflict. Ethology 104, 789-803.

McElligott, A. G., O'Neill, K. P. \& Hayden, T. J. 1999: Cumulative long-term investment in vocalization and mating success of fallow bucks, Dama dama. Anim. Behav. 57, 1159-1167.

McElligott, A. G., Gammell, M. P., Harty, H. C., Paini, D. R., Murphy, D. T., Walsh, J. T. \& Hayden, T. J. 2001: Sexual size dimorphism in fallow deer (Dama dama): do larger, heavier males gain greater mating success? Behav. Ecol. Sociobiol. 49, 266-272.

McElligott, A. G., Naulty, F., Clarke, W. \& Hayden, T. J. 2003: The somatic cost of reproduction: what determines reproductive effort in prime-aged fallow bucks? Evol. Ecol. Res. 5, 1239-1250.

McElligott, A. G., Birrer, M. \& Vannoni, E. 2006. Retraction of the mobile descended larynx during groaning enables fallow bucks (Dama dama) to lower their formant frequencies. J. Zool. (Lond.) $270,340-345$.

McGarigal, K. S., Cushman, S. \& Stafford, S. 2000: Multivariate Statistics for Wildlife and Ecology Research. Springer-Verlag, New York.

Morton, E. S. 1977: On the occurrence and significance of motivation-structural rules in some birds and mammal sounds. Am. Nat. 111, 855-869.

Morton, E. S. 1982: Grading, discreteness, redundancy, and motivational-structural rules. In: Acoustic Communication in Birds (Kroodsma, D. E. \& Miller, E. H., eds). Academic Press, New York 1, pp. 183-212.

O'Connell-Rodwell, C. E., Arnason, B. T. \& Hart, L. A. 2000: Seismic properties of Asian elephant (Elephas maximus) vocalizations and locomotion. J. Acoust. Soc. Am. 108, 3066-3072.

Owren, M. J. \& Linker, C. D. 1995: Some analysis methods that may be useful to acoustic primatologists. In: Current Topics in Primate Vocal Communication. (Zimmermann, E., Newman, J. D. \& Jürgens, U., eds). Plenum Press, New York, pp. 1-27.

Owren, M. J., Seyfarth, R. M. \& Cheney, L. D. 1997: The acoustic features of vowel-like grunt calls in chacma baboons (Papio cynocephalus ursinus): implications for production processes and functions. J. Acoust. Soc. Am. 101, 2951-2963. 
R Development Core Team 2004: R: A Language and Environment for Statistical Computing. R Foundation for Statistical Computing, Vienna, Austria. Available at: http://www.R-project.org.

Reby, D. \& McComb, K. 2003a: Anatomical constraints generate honesty: acoustic cues to age and weight in the roars of red deer stags. Anim. Behav. 65, 519-530.

Reby, D. \& McComb, K. E. 2003b: Vocal communication and reproduction in deer. Adv. Study Behav. 33, 231-264.

Reby, D., Joachim, J., Lauga, J., Lek, S. \& Aulagnier, S. 1998: Individuality in the groans of fallow deer (Dama dama) bucks. J. Zool. (Lond.) 245, 79-84.

Reby, D., Cargnelutti, B., Joachim, J. \& Aulagnier, S. 1999a: Spectral acoustic structure of barking in roe deer (Capreolus capreolus). Sex-, age- and individual-related variations. C. R. Acad. Sci. Paris 322, 271-279.

Reby, D., Cargnelutti, B. \& Hewison, A. J. M. 1999b: Contexts and possible functions of barking in roe deer. Anim. Behav. 57, 1121-1128.

Reby, D., McComb, K. E., Cargnelutti, B., Darwin, C., Fitch, W. T. \& Clutton-Brock, T. H. 2005: Red deer stags use formants as assessment cues during intrasexual agonistic interactions. Proc. R. Soc. Lond. B. 272, 941-947.

Reby, D., André-Obrecht, R., Galinier, A., Farinas, J. \& Cargnelutti, B. 2006. Cepstral coefficients and hidden Markov models reveal idiosyncratic voice characteristics in red deer (Cervus elaphus) stags. J. Acoust. Soc. Am. 120, 4080—4089.

Rendall, D. 2003: Acoustic correlates of caller identity and affect intensity in the vowel-like grunt vocalizations of baboons. J. Acoust. Soc. Am. 113, 3390-3402.

Rendall, D., Rodman, P. S. \& Emond, R. E. 1996: Vocal recognition of individuals and kin in free-ranging rhesus monkeys. Anim. Behav. 51, 1007-1015.

Rendall, D., Owren, M. J. \& Rodman, P. S. 1998: The role of vocal tract filtering in identity cueing in rhesus monkey (Macaca mulatta) vocalizations. J. Acoust. Soc. Am. 103, 602-614.

Riede, T. \& Fitch, W. T. 1999: Vocal tract length and acoustic of vocalization in the domestic dog (Canis familiaris). J. Exp. Biol. 202, 2859-2867.

Riede, T. \& Zuberbühler, K. 2003: Pulse register phonation in Diana monkey alarm calls. J. Acoust. Soc. Am. 113, 2919-2925.

Riede, T., Bronson, E., Hatzikirou, H. \& Züberbühler, K. 2005: Vocal production mechanisms in a nonhuman primate: morphological data and a model. J. Hum. Evol. 48, 85-96.

Sayigh, L. S., Tyack, P. L., Wells, R. S., Solow, A. R., Scott, M. D. \& Irvine, A. B. 1998: Individual recognition in wild bottlenose dolphins: a field test using playback experiments. Anim. Behav. 57, 41-50.

Solow, A. R. 1990: A randomization test for misclassification probability in discriminant analysis. Ecology $71,2379-2382$.

Soltis, J., Leong, K. \& Savage, A. 2005: African elephant vocal communication II: rumble variation reflects the individual identity and emotional state of callers. Anim. Behav. 70, 589—599.

Stoddard, P. K. 1996: Vocal recognition of neighbors by territorial passerines. In: Ecology and Evolution of Acoustic Communication in Birds (Kroodsma, D. E. \& Miller, E. H., eds). Cornell University Press, Ithaca, NY, pp. 356-374.

Titze, I. R. 1994: Principles of Voice Production. Prentice Hall, Englewood Cliffs, NJ.

Titze, I. R. \& Liang, H. 1993: Comparison of F0 extraction methods for high-precision voice perturbation measurements. J. Speech Hear. Res. 36, 1120-1133.

Titze, I. R., Horii, Y. \& Scherer, R. C. 1987: Some technical consideration in voice perturbation measurements. J. Speech Hear. Res. 30, 252-260.

Torriani, M. V. G., Vannoni, E. \& McElligott, A. G. 2006. Mother-young recognition in an ungulate hider species: a unidirectional process. Am. Nat. 168, 412-420. 
Vaňková, D. \& Málek, J. 1997: Characteristics of the vocalizations of red deer (Cervus elaphus) hinds and calves. Bioacoustics 7, 281-289.

Vannoni, E., Torriani, M. V. G. \& McElligott, A. G. 2005: Acoustic signalling in cervids: a methodological approach for measuring vocal communication in fallow deer. Cogn. Brain Behav. 9, 551-566.

Wilden, I., Herzel, H., Peters, G. \& Tembrock, G. 1998: Subharmonics, biphonation, and deterministic chaos in mammal vocalization. Bioacoustics 9, 171-196.

Zimmerman, E. \& Lerch, C. 1993: The complex acoustic design of an advertisement call in male mouse lemurs (Microcebus murinus, Prosimii, Primates) and source of its variation. Ethology 93, 211224. 